\title{
Solvent-free Synthesis of Propargylic Alcohols using ZnO as a New and Reusable Catalyst by Direct Addition of Alkynes to Aldehydes
}

\author{
Mona Hosseini-Sarvari* and Zahra Mardaneh \\ Department of Chemistry, Faculty of Science, Shiraz University, Shiraz 71454, I. R. Iran. *E-mail: hossaini@shirazu.ac.ir \\ Received August 22, 2011, Accepted October 11, 2011
}

\begin{abstract}
Under solvent-free conditions, the synthesis of propargylic alcohols by direct addition of terminal alkynes to aldehydes promoted by $\mathrm{ZnO}$ as a novel, commercially, and cheap catalyst is described. Furthermore, the catalyst can be reused for several times without any significant loss of its catalytic activity.
\end{abstract}

Key Words : Propargylic alcohols, ZnO, Terminal alkynes, Solvent-free conditions, Aldehydes

\section{Introduction}

Carbon-carbon bond formation reactions are amongst the important transformations in organic chemistry. The alkynylation of carbonyl compounds is a direct chain extension reaction. ${ }^{1-8}$ Propargylic alcohols are important and versatile building blocks for many biologically active compounds and natural products such as adociacetylene $\mathrm{B},{ }^{9}$ longimicin $\mathrm{D},{ }^{10}$ leukotriene $\mathrm{B}_{4},{ }^{11}$ steroid, ${ }^{12}$ prostaglandins, ${ }^{13}$ and carotenoids, ${ }^{14}$ and have gained considerable attention in recent years. The most common methods to obtain propargylic alcohols are the nucleophilic addition of stoichiometric amounts of strong bases such as organolithium, dialkyl zinc or organomagnesium reagents with or without Lewis acids. ${ }^{15-22}$ However, the use of stoichiometric amounts of metal reagents, and a separate step for metal acetylide preparation make it difficult to achieve an atom-economical process with high total efficiency. Several improved procedures including addition of alkynyl-B, ${ }^{23} \mathrm{Al},{ }^{24,25} \mathrm{~V}^{26}$ and $\mathrm{Ce}^{27}$ to carbonyl compounds represents a useful method for producing these compounds. However, these methods are not straightforward.

The in situ catalytic generation of metal acetylide and their use in addition reaction to carbonyl compounds is a good choice for this purpose. Recently, considerable progress has been developed in the alkynylation of carbonyl compounds using a Lewis acids such as $\mathrm{Zn}(\mathrm{OTf})_{2}, \mathrm{Sn}(\mathrm{OTf})_{2},{ }^{28-34} \mathrm{GaI}_{3},{ }^{35}$ $\mathrm{ZnCl}_{2},{ }^{36}$ indium, ${ }^{37-41}{ }^{i} \mathrm{PrMgBr}^{42} \mathrm{CrCl}_{3}{ }^{43}$ and $\mathrm{Me}_{3} \mathrm{Ga}^{44}$ combined with a Lewis base. Haloalkynes also form good substrates for metalation with metals such as $\mathrm{Zn}$ followed by the nucleophilic addition onto the carbonyl compounds. ${ }^{45-48}$ Although the alkynylation of carbonyl compounds have advantages, but the substrate generality in many procedures are quite limited due mainly to high temperature or strongly basic conditions. Moreover, in most methods, the reaction times are long, the yields are low, the uses of strong bases are needed, and the corresponding procedures require harmful organic solvents, making them unfavorable procedures.

Despite the development of a number of Lewis acid and bases systems for the alkynylation of aldehydes, to the best of our knowledge, no attention was paid to a method for direct addition of alkynes to aldehydes without use of any active metals, solvents, and strong bases. Therefore, because of the importance of propargylic alcohols both in academic and industrial applications, it provides the constant driving force for the development of this reaction.

An important goal in modern synthetic methodology is the development of processes that are facile to carry out in the laboratory without recourse to inert atmosphere or limited using solvents especially toxic organic solvents. Therefore, the quest for cheap, environmentally friendly catalysts and mild reaction conditions is still a major challenge.

$\mathrm{ZnO}$ is a 'green', cheap and efficient catalyst in modern organic synthesis. It has become the focus of attention in several environmentally friendly and atom-economical organic transformations. Over the course of our continuing studies on $\mathrm{ZnO}$ as catalyst, ${ }^{49}$ we herein wish to report the novel synthesis of propargylic alcohols by direct addition of terminal alkynes to aldehydes in solvent- and base-free conditions. Very simple reaction conditions and environmentally friendly catalyst make this transformation an attractive option for the straightforward preparation of propargylic alcohols.

\section{Experimental Section}

Commercial solid $\mathrm{ZnO}\left(\mathrm{SA}: \sim 5 \mathrm{~m}^{2} / \mathrm{g}\right.$ ) was purchased from Fluka no. 89490. ${ }^{1} \mathrm{H}$ NMR and ${ }^{13} \mathrm{C}$ NMR spectra measured on Bruker Advance DPX FT 250 and $62.9 \mathrm{MHz}$ spectrometry with TMS as an internal standard. IR spectra obtained on a Perkin-Elmer or FTIR-800 instruments. Mass spectra were obtained on a Shimadzu GCMS0QP 1000EX at 20 and/or $70 \mathrm{eV}$. Elemental analyses performed on Thermo Finnigan, Flash EA 1112 series microanalyzer by the head of the CHN lab.

General Procedure. To a mixture of $\mathrm{ZnO}(0.016 \mathrm{~g}, 5$ mol \%) and 1-Alkyne $(1 \mathrm{mmol})$, in an oil bath at $120{ }^{\circ} \mathrm{C}$ and under solvent-free conditions, aldehydes $(1 \mathrm{mmol})$ were added drop-wise. Then the reaction mixture was stirred for a certain period of time as required to complete the reaction (monitored by TLC). The crude reaction mixture was extracted with EtOAc $(3 \times 15 \mathrm{~mL})$. The organic phase were dried over $\mathrm{CaCl}_{2}$ and evaporated, and the resulting crude 
material was purified by column chromatography (hexan/ EtOAc), which afforded the pure propargylic alcohols derivatives. All products were characterized by NMR, IR and mass spectral data which for known compounds were found to be identical with those described in the literature and only ${ }^{1} \mathrm{H}$ and ${ }^{13} \mathrm{C}$ NMR are shown and for new compounds the complete spectroscopic data are described as bellow.

Recycling of the Catalyst. Upon completion, the reaction of isobutyraldehyde 1a with phenylacetylene $\mathbf{2 a}$, the reaction mixture was diluted with EtOAc, centrifuged and the $\mathrm{ZnO}$ was separated, washed with EtOAc and water, dried in an oven at $100{ }^{\circ} \mathrm{C}$, and reused for the similar reaction. The recycling of the catalyst was repeated for four runs without appreciable loss of its catalytic activity (Table 3 ).

\section{Spectral Data.}

4-Methyl-1-phenylpent-1-yn-3-ol (3a): ${ }^{16 e}$ Pale yellow oil: ${ }^{1} \mathrm{H}$ NMR (250 MHz, $\left.\mathrm{CDCl}_{3}\right): \delta$ 7.32-7.36 (m, 2H, Ar-H), 7.19-7.22 (m, 3H, Ar-H), 4.29 (d, $J=5.64$ Hz, 1H, -C$(\mathrm{OH})-$ ), 2.33 (br, 1H, -OH), 1.84-1.89 (m, 1H, - $\left.\underline{\mathrm{H}}\left(\mathrm{CH}_{3}\right)_{2}-\right), 0.95$ (d, $J=6.66 \mathrm{~Hz}, 6 \mathrm{H},-\mathrm{Me}) ;{ }^{13} \mathrm{C} \mathrm{NMR}\left(62.9 \mathrm{MHz}, \mathrm{CDCl}_{3}\right): \delta$ 131.6, 128.24, 128.23, 122.79, 89.0, 85.4, 68.2, 34.6, 18.2, 17.5 .

1-Phenylpent-1-yn-3-ol (3b): ${ }^{16 e}$ Pale yellow oil: ${ }^{1} \mathrm{H}$ NMR (250 MHz, $\left.\mathrm{CDCl}_{3}\right): \delta$ 7.41-7.45 (m, 2H, Ar-H), 7.28-7.32 (m, 3H, Ar-H), 4.56 (t, $J=6.42 \mathrm{~Hz}, 1 \mathrm{H},-\mathrm{CH}(\mathrm{OH})-), 2.27$ (br, $1 \mathrm{H},-\mathrm{OH}), 1.76-1.88$ (m, $\left.2 \mathrm{H},-\mathrm{CH}(\mathrm{OH})-\mathrm{CH}_{2}-\right), 1.08-1.04$ (t, $J=7.32 \mathrm{~Hz}, 3 \mathrm{H},-\mathrm{Me}) ;{ }^{13} \mathrm{C} \mathrm{NMR}\left(62.9 \mathrm{MHz}, \mathrm{CDCl}_{3}\right): \delta$ 131.6, 128.3, 128.2, 122.6, 90.02, 84.8, 64.1, 30.9, 9.5.

1-Phenylhex-1-yn-3-ol (3c): ${ }^{22}$ Pale yellow oil: ${ }^{1} \mathrm{H}$ NMR $\left(250 \mathrm{MHz}, \mathrm{CDCl}_{3}\right): \delta$ 7.12-7.15 (m, 2H, Ar-H), 6.98-7.06 (m, 3H, Ar-H), 4.30-4.33 (t, $J=6.52 \mathrm{~Hz}, 1 \mathrm{H},-\mathrm{C} \underline{\mathrm{H}}(\mathrm{OH})-)$, 2.04 (br, $1 \mathrm{H},-\mathrm{OH}), 1.32-1.39\left(\mathrm{~m}, 2 \mathrm{H},-\mathrm{CH}_{2}-\mathrm{CH}_{2}-\mathrm{CH}_{3}\right)$, 1.17-1.22 (m, 2H, $\left.-\mathrm{CH}_{2}-\mathrm{CH}_{2}-\mathrm{CH}_{3}\right), 0.62-0.70(\mathrm{t}, J=6.71$ $\left.\mathrm{Hz}, 3 \mathrm{H},-\mathrm{CH}_{2}-\mathrm{CH}_{2}-\mathrm{CH}_{3}\right) ;{ }^{13} \mathrm{C} \mathrm{NMR}\left(62.9 \mathrm{MHz}, \mathrm{CDCl}_{3}\right): \delta$ 131.6, 129.8, 128.1, 126.6, 90.40, 84.4, 63.5, 36.06,18.4, 13.9.

1-Phenylhept-1-yn-3-ol (3d): Pale yellow oil: ${ }^{1} \mathrm{H}$ NMR $\left(250 \mathrm{MHz}, \mathrm{CDCl}_{3}\right): \delta$ 7.39-7.43 (m, 2H, Ar-H), 7.27-7.31 (m, 3H, Ar-H ), 4.57 (t, $J=6.45 \mathrm{~Hz}, 1 \mathrm{H},-\mathrm{CH}(\mathrm{OH})-), 2.27$ (br, 1H, -OH), 1.29-1.38 (m, 6H, - $\left.\left(\mathrm{CH}_{2}\right)_{3}-\mathrm{CH}_{3}\right), 0.87-0.93$ (t, $J=5.71 \mathrm{~Hz}, 3 \mathrm{H},-\mathrm{Me}) ;{ }^{13} \mathrm{C}$ NMR $\left(62.9 \mathrm{MHz}, \mathrm{CDCl}_{3}\right): \delta$ 131.6, 128.27, 128.22, 126.7, 90.3, 84.6, 64.9, 42.6, 27.7, 22.6, 14.0. EI-MS: $m / z 188\left(\mathrm{M}^{+}\right)$. Anal. Calcd for molecular formula $\mathrm{C}_{13} \mathrm{H}_{16} \mathrm{O}: \mathrm{C}, 82.94 ; \mathrm{H}, 8.57 \%$; found: $\mathrm{C}, 82.86, \mathrm{H}$, $8.48 \%$.

4-Ethyl-1-phenyloct-1-yn-3-ol (3e): Pale yellow oil: ${ }^{1} \mathrm{H}$ NMR (250 MHz, $\left.\mathrm{CDCl}_{3}\right): \delta$ 7.31-7.36 (m, 2H, Ar-H), 7.19$7.24(\mathrm{~m}, 3 \mathrm{H}, \mathrm{Ar}-\mathrm{H}), 4.52(\mathrm{t}, J=6.52 \mathrm{~Hz}, 1 \mathrm{H},-\mathrm{CH}(\mathrm{OH}))$, 2.25 (br, 1H, -OH), 2.19-2.23 (m, 1H, -Cㅌ-) 1.35-1.59 (m, $\left.8 \mathrm{H},-\mathrm{CH}\left(\mathrm{C}_{2}-\mathrm{CH}_{3}\right)\left(\mathrm{C}_{2} \underline{\mathrm{H}}_{6} \mathrm{CH}_{3}\right)\right), 0.82-0.90(\mathrm{~m}, 6 \mathrm{H}, \mathrm{Me}) ;{ }^{13} \mathrm{C}$ NMR $\left(62.9 \mathrm{MHz}, \mathrm{CDCl}_{3}\right): \delta 131.6,128.27,127.5,126.1$, 121.2, 90.7, 84.7, 65.3 , 45.2, 31.3 , 30.7, 21.7, 14.1, 11.8. EIMS: $\mathrm{m} / z 230\left(\mathrm{M}^{+}\right)$. Anal. Calcd for molecular formula $\mathrm{C}_{16} \mathrm{H}_{22} \mathrm{O}$ : C, 83.43; H, 9.63\%; found: C, 83.37, H, 9.58\%.

1,3-Diphenylprop-2-yn-1-ol (3f): ${ }^{16 e}$ Pale yellow oil: ${ }^{1} \mathrm{H}$ NMR (250 MHz, $\left.\mathrm{CDCl}_{3}\right): \delta$ 7.46-7.50 (m, 2H, Ar-H), 7.32-
7.36 (m, 2H, Ar-H), 7.23-7.28 (m, 2H, Ar-H), 7.14-7.19 (m, $4 \mathrm{H}, \mathrm{Ar}-\mathrm{H}), 5.54$ (s, 1H, $-\mathrm{CH}(\mathrm{OH})), 2.72(\mathrm{br}, 1 \mathrm{H},-\mathrm{OH}) ;{ }^{13} \mathrm{C}$ NMR $\left(62.9 \mathrm{MHz}, \mathrm{CDCl}_{3}\right): \delta 140.7,131.8,129.9,128.7$, $128.4,127.8,126.8,122.5,88.9,86.6,65.0$.

1-(4-Nitrophenyl)-3-phenylprop-2-yn-1-ol $\quad(3 g):^{14} \quad{ }^{1} \mathrm{H}$ NMR (250 MHz, $\left.\mathrm{CDCl}_{3}\right) \delta 8.10(\mathrm{~d}, 2 \mathrm{H}, J=7.5 \mathrm{~Hz}, \mathrm{Ar}-\mathrm{H})$; $7.64(\mathrm{~d}, 2 \mathrm{H}, J=7.5 \mathrm{~Hz}, \mathrm{Ar}-\mathrm{H}), 7.36$ (d, 2H, $J=7.5 \mathrm{~Hz}$, ArH), 7.25-7.30 (m, 3H, Ar-H), $5.72(\mathrm{~s}, 1 \mathrm{H},-\mathrm{CH}(\mathrm{OH})), 2.56$ (br s, $1 \mathrm{H}),{ }^{13} \mathrm{C}$ NMR (62.9 MHz, $\left.\mathrm{CDCl}_{3}\right) \delta$ 141.6, 140.4, 131.6, 128.9, 128.3, 127.3, 123.7, 121.6, 87.4, 87.5, 63.8.

1-(4-Chlorophenyl)-3-phenylprop-2-yn-1-ol (3h): ${ }^{14}{ }^{1} \mathrm{H}$ $\mathrm{NMR}\left(250 \mathrm{MHz}, \mathrm{CDCl}_{3}\right) \delta 7.76(\mathrm{~d}, 2 \mathrm{H}, J=7.5 \mathrm{~Hz}, \mathrm{Ar}-\mathrm{H})$; 7.24 (d, 2H, $J=7.5 \mathrm{~Hz}$, Ar-H), 7.10-7.21 (m, 5H, Ar-H), $5.52(\mathrm{~s}, 1 \mathrm{H},-\mathrm{CH}(\mathrm{OH})), 2.26$ (br s, $1 \mathrm{H}),{ }^{13} \mathrm{C}$ NMR $(62.9$ $\left.\mathrm{MHz}, \mathrm{CDCl}_{3}\right) \delta 139.6,133.6,131.7,128.8,128.3,128.2$, $127.8,122.7,88.4,86.5,64.8$.

1-(4-Methylphenyl)-3-phenylprop-2-yn-1-ol $\quad(3 \mathrm{ii}):{ }^{15}{ }^{1} \mathrm{H}$ NMR (250 MHz, $\left.\mathrm{CDCl}_{3}\right) \delta 7.63$ (d, 2H, J=7.5 Hz, Ar-H); 7.46 (d, 2H, $J=7.5 \mathrm{~Hz}, \mathrm{Ar}-\mathrm{H}), 7.34$ (d, 2H, $J=7.5 \mathrm{~Hz}$, ArH), 7.23-7.29 (m, 3H, Ar-H), $5.51(\mathrm{~s}, 1 \mathrm{H},-\mathrm{CH}(\mathrm{OH})), 2.43$ (s, $3 \mathrm{H},-\mathrm{Me}), 2.20$ (br s, $1 \mathrm{H}),{ }^{13} \mathrm{C} \mathrm{NMR}\left(62.9 \mathrm{MHz}, \mathrm{CDCl}_{3}\right) \delta$ 138.7, 137.4, 131.2, 129.6, 128.6, 128.1, 127.2, 122.4, 88.3, $86.4,64.6,20.8$.

4-(1-Hydroxy-3-phenylprop-2-ynyl)benzonitrile (3j): ${ }^{36}$ ${ }^{1} \mathrm{H}$ NMR $\left(250 \mathrm{MHz}, \mathrm{CDCl}_{3}\right) \delta 7.71(\mathrm{~d}, 2 \mathrm{H}, J=8.0 \mathrm{~Hz}, \mathrm{Ar}-$ $\mathrm{H}), 7.63$ (d, 2H, $J=8.0 \mathrm{~Hz}, \operatorname{Ar}-\mathrm{H}), 7.42$ (d, 2H, $J=7.8 \mathrm{~Hz}$, Ar-H), 7.27-7.35 (m, 3H, Ar-H), 5.64 (s, 1H, $-\mathrm{CH}(\mathrm{OH})$ ), 2.53 (br s, $1 \mathrm{H}),{ }^{13} \mathrm{C} \mathrm{NMR}\left(62.9 \mathrm{MHz}, \mathrm{CDCl}_{3}\right) \delta 143.6$, $136.3,131.3,129.5,128.3,127.1,121.7,118.5,111.7,87.5$, 87.2, 64.0.

1-(4-Methoxyphenyl)-3-phenylprop-2-yn-1-ol (3k): ${ }^{36}$ ${ }^{1} \mathrm{H}$ NMR $\left(250 \mathrm{MHz}, \mathrm{CDCl}_{3}\right) \delta 7.45$ (d, 2H, $J=8.0 \mathrm{~Hz}$, Ar$\mathrm{H}), 7.38$ (d, 2H, J=8.0 Hz, Ar-H), 7.29-7.30 (m, 3H, Ar-H), 6.89 (d, 2H, J=8.0 Hz, Ar-H), 5.60 (s, 1H, - $\underline{\mathrm{H}}(\mathrm{OH})), 3.780$ (s, 3H, -OMe), 2.49 (br s, 1H), ${ }^{13} \mathrm{C} \mathrm{NMR}\left(62.9 \mathrm{MHz}, \mathrm{CDCl}_{3}\right)$ $\delta 158.7,133.4,131.1,128.7,128.6,128.2,122.8,114.1$, $88.3,86.5,64.1,55.2$.

1-(4-Nitrophenyl)-3-phenylprop-2-yn-1-ol $\quad(3 \mathrm{I}):{ }^{35}{ }^{1} \mathrm{H}$ NMR $\left(250 \mathrm{MHz}, \mathrm{CDCl}_{3}\right) \delta 8.10(\mathrm{~d}, 2 \mathrm{H}, J=7.5 \mathrm{~Hz}, \mathrm{Ar}-\mathrm{H})$, 7.67 (d, 2H, $J=7.5 \mathrm{~Hz}, \mathrm{Ar}-\mathrm{H}), 7.49$ (d, 2H, $J=7.5 \mathrm{~Hz}, \mathrm{Ar}-$ $\mathrm{H}), 7.42-7.30$ (m, 3H, Ar-H), 5.67 (s, 1H, - $\underline{\mathrm{H}}(\mathrm{OH})), 2.63$ (br s, $1 \mathrm{H}) ;{ }^{13} \mathrm{C}$ NMR $\left(62.9 \mathrm{MHz}, \mathrm{CDCl}_{3}\right) \delta 147.3,146.4$, 131.2, 128.1, 127.3, 127.0, 124.7, 121.1, 87.8, 87.1, 63.4.

1-(Naphthalen-2-yl)-3-phenylprop-2-yn-1-ol (3m): ${ }^{35} \mathrm{H}$ NMR $\left(250 \mathrm{MHz}, \mathrm{CDCl}_{3}\right) \delta 8.31(\mathrm{~d}, 1 \mathrm{H}, J=7.5 \mathrm{~Hz}, \mathrm{Ar}-\mathrm{H})$, 7.71-7.90 (m, 3H, Ar-H), 7.37-7.46 (m, 5H, Ar-H), 7.267.30 (m, 3H, Ar-H), 5.33 (s, 1H, - $\underline{\mathrm{H}}(\mathrm{OH})), 2.56$ (br s, 1H), ${ }^{13} \mathrm{C}$ NMR $\left(62.9 \mathrm{MHz}, \mathrm{CDCl}_{3}\right)$ 134.8, 134.0, 131.2, 130.8, $129.0,128.4,128.3,128.1,125.8,125.2,124.7,124.3$, $123.5,122.2,88.6,86.8,63.5$.

3-Phenyl-1-(pyridin-3-yl)prop-2-yn-1-ol (3n): ${ }^{36}{ }^{1} \mathrm{HNMR}$ $\left(250 \mathrm{MHz}, \mathrm{CDCl}_{3}\right) \delta 8.78(\mathrm{~d}, 1 \mathrm{H}, J=8.0 \mathrm{~Hz}, \mathrm{Ar}-\mathrm{H}), 8.60(\mathrm{~d}$, $1 \mathrm{H}, J=8.0 \mathrm{~Hz}, \mathrm{Ar}-\mathrm{H}), 7.89$ (d, 1H, $J=8.0 \mathrm{~Hz}, \mathrm{Ar}-\mathrm{H}), 7.51-$ 7.58 (m, 2H, Ar-H), 7.34-7.40 (m, 4H, Ar-H), 5.57 (s, 1H, $-\mathrm{CH}(\mathrm{OH})), 2.53$ (br s, 1H), ${ }^{13} \mathrm{C}$ NMR $\left(62.9 \mathrm{MHz}, \mathrm{CDCl}_{3}\right) \delta$ $148.0,147.4,136.8,134.2,131.3,128.2,127.5,123.2$, $122.7,88.4,86.7,62.8$. 
2-Methyltridec-4-yn-3-ol (3o): Pale yellow oil: ${ }^{1} \mathrm{H}$ NMR $\left(250 \mathrm{MHz}, \mathrm{CDCl}_{3}\right): \delta 4.76-4.77$ (d, $J=5.5 \mathrm{~Hz}, 1 \mathrm{H},-\mathrm{C} \underline{\mathrm{H}}(\mathrm{OH})$ ), 2.31 (br, $1 \mathrm{H},-\mathrm{OH}), 1.17-1.55$ (m, $\left.15 \mathrm{H},-\mathrm{CH}_{2}-\right), 0.81-0.98$ (m, 9H, Me); ${ }^{13} \mathrm{C}$ NMR $\left(62.9 \mathrm{MHz}, \mathrm{CDCl}_{3}\right): \delta 83.2,80.55$, 69.0, 34.5, 31.8, 29.3, 29.1, 28.5, 27.6, 22.6, 18.6, 17.6, 14.08. EI-MS: $m / z 210\left(\mathrm{M}^{+}\right)$. Anal. Calcd for molecular formula $\mathrm{C}_{14} \mathrm{H}_{26} \mathrm{O}$ : C, 79.94; $\mathrm{H}, 12.46 \%$; found: $\mathrm{C}, 79.82, \mathrm{H}$, $12.33 \%$.

2-Methyl-6-(1-naphthyloxy)hex-4-yn-3-ol (3p): Pale yellow oil: ${ }^{1} \mathrm{H}$ NMR (250 MHz, $\left.\mathrm{CDCl}_{3}\right): \delta 8.76$ (d, $J=7.05$ $\mathrm{Hz}, 1 \mathrm{H}, \mathrm{Ar}-\mathrm{H}), 8.06$ (d, $J=6.75 \mathrm{~Hz}, 1 \mathrm{H}, \mathrm{Ar}-\mathrm{H}), 7.73-7.82$ (m, 3H, Ar-H), 7.54-7.62 (m, 1H, Ar-H), 6.99 (d, $J=6.61$ $\mathrm{Hz}, 1 \mathrm{H}, \mathrm{Ar}-\mathrm{H}), 4.85$ (s, $\left.2 \mathrm{H},-\mathrm{O}-\mathrm{C}_{2}{ }_{2}\right), 4.36$ (d, $J=4.76 \mathrm{~Hz}$, $1 \mathrm{H},-\mathrm{CH}(\mathrm{OH})), 2.92(\mathrm{br}, 1 \mathrm{H},-\mathrm{OH}), 1.85-1.88(\mathrm{~m}, 1 \mathrm{H}$, $\left.-\mathrm{C} \underline{\mathrm{H}}\left(\mathrm{CH}_{3}\right)_{2}\right), 0.97(\mathrm{~d}, J=6.5 \mathrm{~Hz}, 6 \mathrm{H}, \mathrm{Me}) ;{ }^{13} \mathrm{C}$ NMR $(62.9$ $\left.\mathrm{MHz}, \mathrm{CDCl}_{3}\right): \delta 154.2,135.5,127.0,126.4,125.7,124.9$, $122.3,121.08,121.07,109.4,86.1,85.6,68.1,56.3,34.3$, 17.6. EI-MS: $m / z 254\left(\mathrm{M}^{+}\right)$; Anal. Calcd for molecular formula $\mathrm{C}_{17} \mathrm{H}_{18} \mathrm{O}_{2}$ : C, $80.28 ; \mathrm{H}, 7.13 \%$; found: $\mathrm{C}, 80.13, \mathrm{H}$, $7.04 \%$.

2-Methyl-6-(p-tolyloxy)hex-4-yn-3-ol (3q): Pale yellow oil: IR (neat): $v$ 3487, 2966, 2934, 2289, $1237 \mathrm{~cm}^{-1} .{ }^{1} \mathrm{H}$ NMR $\left(250 \mathrm{MHz}, \mathrm{CDCl}_{3}\right): \delta^{1} \mathrm{H} \mathrm{NMR}^{2}\left(250 \mathrm{MHz}, \mathrm{CDCl}_{3}\right): \delta$ 7.25 (d, $J=7.90 \mathrm{~Hz}, 2 \mathrm{H}, \mathrm{Ar}-\mathrm{H}), 6.89(\mathrm{~d}, J=8.50 \mathrm{~Hz}, 2 \mathrm{H}$, Ar-H), $4.68\left(\mathrm{~s}, 2 \mathrm{H},-\mathrm{OCH}_{2}-\right), 4.11(\mathrm{~d}, J=5.42 \mathrm{~Hz}, 1 \mathrm{H}$, $-\mathrm{C} \underline{\mathrm{H}}(\mathrm{OH})), 2.6(\mathrm{br}, 1 \mathrm{H},-\mathrm{OH}), 1.93(\mathrm{~m}, J=7.60 \mathrm{~Hz}, 1 \mathrm{H}$, $\left.-\mathrm{C} \underline{\mathrm{H}}\left(\mathrm{CH}_{3}\right)_{2}\right), 2.21(\mathrm{~s}, 3 \mathrm{H},-\mathrm{Me}), 0.83(\mathrm{~d}, J=6.60 \mathrm{~Hz}, 6 \mathrm{H}$, -Me); ${ }^{13} \mathrm{C}$ NMR $\left(62.9 \mathrm{MHz}, \mathrm{CDCl}_{3}\right): \delta 155.8,131.6,128.3$, 117.6, 90.1, 86.1, 61.9, 45.9, 33.6, 20.9, 18.5, 18.4. EI-MS: $\mathrm{m} / z 218\left(\mathrm{M}^{+}\right)$. Anal. Calcd for molecular formula $\mathrm{C}_{14} \mathrm{H}_{18} \mathrm{O}_{2}$ : C, 77.03 ; H, 8.31\%; found: C, 76.92, H, 8.24\%.

6-(4-Chlorophenoxy)-2-methylhex-4-yn-3-ol (3r): Pale yellow oil: IR (neat): $v 3452,2935,2875,2298,1245 \mathrm{~cm}^{-1}$. ${ }^{1} \mathrm{H} \mathrm{NMR}\left(250 \mathrm{MHz}, \mathrm{CDCl}_{3}\right): 7.02$ (d, $\left.J=7.9 \mathrm{~Hz}, 2 \mathrm{H}, \mathrm{Ar}-\mathrm{H}\right)$, $6.81(\mathrm{~d}, J=7.9 \mathrm{~Hz}, 2 \mathrm{H}, \mathrm{Ar}-\mathrm{H}), 4.61\left(\mathrm{~s}, 2 \mathrm{H},-\mathrm{O}-\mathrm{C}_{2}-\right), 4.01$ $(\mathrm{d}, J=6.80 \mathrm{~Hz}, 1 \mathrm{H},-\mathrm{CH}(\mathrm{OH})), 2.93(\mathrm{br}, 1 \mathrm{H},-\mathrm{OH}), 2.18-$ $2.21\left(\mathrm{~m}, 1 \mathrm{H},-\mathrm{C} \underline{\mathrm{H}}\left(\mathrm{CH}_{3}\right)_{2}\right), 0.78-0.94$ (m, 6H, -Me); ${ }^{13} \mathrm{C}$ NMR (62.9 MHz, $\left.\mathrm{DCl}_{3}\right): \delta 156.1,129.6,128.2,116.6,17.3,116.2$, 79.4, 79.2, 69.8, 56.3, 34.3, 17.6; EI-MS: $m / z 238\left(\mathrm{M}^{+}\right)$. Anal. Calcd for molecular formula $\mathrm{C}_{13} \mathrm{H}_{15} \mathrm{ClO}_{2}: \mathrm{C}, 65.41 ; \mathrm{H}$, $6.33 \%$; found: C, $65.36, \mathrm{H}, 6.24 \%$.

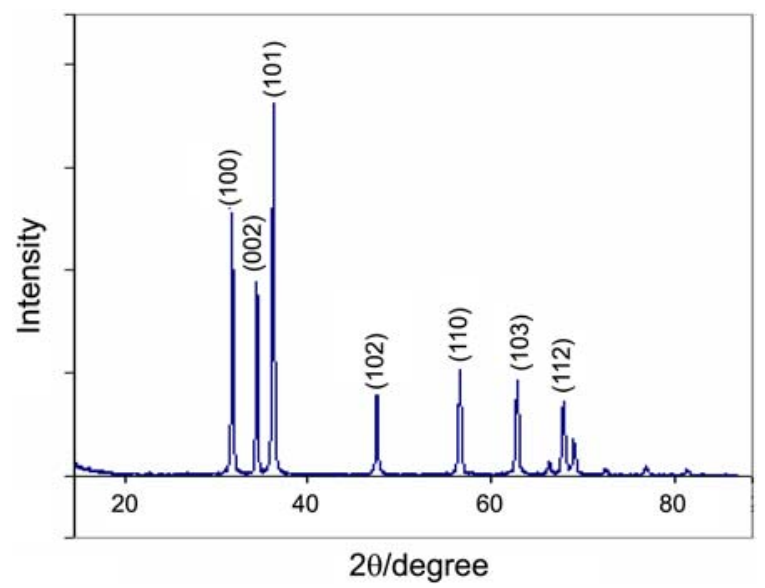

Figure 1. The XRD patterns of $\mathrm{ZnO}$.
1-(4-Chlorophenoxy)-4-(naphthalen-2-yloxy)but-2-yn1-ol (3s): Yellow solid: mp. $>300{ }^{\circ} \mathrm{C} .{ }^{1} \mathrm{H}$ NMR $(250 \mathrm{MHz}$, $\mathrm{CDCl}_{3}$ ): 8.12 (d, $\left.J=7.15 \mathrm{~Hz}, 1 \mathrm{H}, \mathrm{Ar}-\mathrm{H}\right), 7.89$ (d, $J=7.18$ $\mathrm{Hz}, 1 \mathrm{H}, \mathrm{Ar}-\mathrm{H}), 6.89-7.51$ (m, 6H, Ar-H), 6.71 (d, $J=7.20$ $\mathrm{Hz}, \mathrm{Ar}-\mathrm{H}), 4.56$ (s, $\left.2 \mathrm{H},-\mathrm{O}-\mathrm{C}_{2}-\right), 4.20(\mathrm{~s}, 1 \mathrm{H},-\mathrm{CH}(\mathrm{OH}))$, $2.84(\mathrm{br}, 1 \mathrm{H},-\mathrm{OH}) ;{ }^{13} \mathrm{C}$ NMR $\left(62.9 \mathrm{MHz}, \mathrm{DCl}_{3}\right): \bar{\delta} 156.2$, $139.5,135.4,133.6,131.6,128.9,128.4,127.1,126.3$, 124.7, 122.1, 121.1, 121.05, 109.5, 86.1, 85.5, 68.2, 56.2; EI-MS: $m / z 322\left(\mathrm{M}^{+}\right)$. Anal. Calcd for molecular formula $\mathrm{C}_{20} \mathrm{H}_{15} \mathrm{ClO}_{2}$ : C, 74.42 ; $\mathrm{H}, 4.68 \%$; found: $\mathrm{C}, 74.35$, $\mathrm{H}, 4.55 \%$.

\section{Results and Discussion}

Initially, the solid $\mathrm{ZnO}$ (Fluka no. 89490), which was used in this reaction was characterized by X-ray diffraction

Table 1. Optimization of the synthesis of 1,3-diphenylprop-2-yn-1ol 3a using $\mathrm{ZnO}^{a}$

\begin{tabular}{|c|c|c|c|c|c|c|}
\hline Entry & $\begin{array}{c}\text { Phenyl } \\
\text { acetylene } \\
\text { 2a (equiv) }\end{array}$ & $\begin{array}{c}\text { Catalyst } \\
(\mathrm{mol} \%)\end{array}$ & Solvent ${ }^{b}$ & Base $^{c}$ & $\begin{array}{l}\text { Time } \\
\text { (h) }\end{array}$ & $\begin{array}{l}\text { Yield } \\
(\%)^{d}\end{array}$ \\
\hline 1 & 1 & $\mathrm{ZnO}(5)$ & none & none & 24 & 50 \\
\hline 2 & 1.5 & $\mathrm{ZnO}(5)$ & none & none & 24 & 50 \\
\hline 3 & 2 & $\mathrm{ZnO}(5)$ & none & none & 7 & 98 \\
\hline 4 & 2.5 & $\mathrm{ZnO}(5)$ & none & none & 7 & 98 \\
\hline 5 & 1 & $\mathrm{ZnO}(5)$ & none & none & 7.5 & $98^{e}$ \\
\hline 6 & 1 & $\mathrm{ZnO}(5)$ & $\mathrm{MeOH}$ & none & 24 & $\operatorname{trace}^{e}$ \\
\hline 7 & 1 & $\mathrm{ZnO}(5)$ & THF & none & 24 & $\operatorname{trace}^{e}$ \\
\hline 8 & 1 & $\mathrm{ZnO}(5)$ & DMF & none & 24 & $\operatorname{trace}^{e}$ \\
\hline 9 & 1 & $\mathrm{ZnO}(5)$ & $\mathrm{CH}_{3} \mathrm{CN}$ & none & 24 & trace $^{e}$ \\
\hline 10 & 1 & $\mathrm{ZnO}(5)$ & PEG-300 & none & 24 & $\operatorname{trace}^{e}$ \\
\hline 11 & 1 & $\mathrm{ZnO}(5)$ & $\mathrm{CH}_{2} \mathrm{Cl}_{2}$ & none & 24 & $\operatorname{trace}^{e}$ \\
\hline 12 & 1 & $\mathrm{ZnO}(5)$ & Toluene & none & 24 & $\operatorname{trace}^{e}$ \\
\hline 13 & 1 & $\mathrm{ZnO}(5)$ & none & $\mathrm{K}_{2} \mathrm{CO}_{3}$ & 12 & $\operatorname{trace}^{e}$ \\
\hline 14 & 1 & $\mathrm{ZnO}(5)$ & none & $\mathrm{Na}_{2} \mathrm{CO}_{3}$ & 12 & $\operatorname{trace}^{e}$ \\
\hline 15 & 1 & $\mathrm{ZnO}(5)$ & none & $\mathrm{K}_{3} \mathrm{PO}_{4}$ & 24 & $\operatorname{trace}^{e}$ \\
\hline 16 & 1 & $\mathrm{ZnO}(5)$ & none & $\mathrm{Cs}_{2} \mathrm{CO}_{3}$ & 24 & $\operatorname{trace}^{e}$ \\
\hline 17 & 1 & $\mathrm{ZnO}(5)$ & none & $\mathrm{Et}_{3} \mathrm{~N}$ & 12 & $\operatorname{trace}^{e}$ \\
\hline 18 & 1 & $\mathrm{ZnO}(5)$ & Toluene & $\mathrm{Et}_{3} \mathrm{~N}$ & 24 & trace $^{e}$ \\
\hline 19 & 1 & $\mathrm{ZnO}(5)$ & $\mathrm{CH}_{2} \mathrm{Cl}_{2}$ & $\mathrm{Et}_{3} \mathrm{~N}$ & 24 & $\operatorname{trace}^{e}$ \\
\hline 20 & 1 & none & none & none & 24 & $0^{e}$ \\
\hline 21 & 1 & $\mathrm{ZnO}(20)$ & none & none & 7 & $98^{e}$ \\
\hline 22 & 1 & $\mathrm{ZnO}(15)$ & none & none & 7 & $98^{e}$ \\
\hline 23 & 1 & $\mathrm{ZnO}(10)$ & none & none & 7 & $98^{e}$ \\
\hline 24 & 1 & $\mathrm{ZnO}(5)$ & none & none & 7.5 & $98^{e}$ \\
\hline 25 & 1 & $\mathrm{ZnO}(4)$ & none & none & 10 & $90^{e}$ \\
\hline 26 & 1 & $\mathrm{ZnO}(2)$ & none & none & 24 & $85^{e}$ \\
\hline 27 & 1 & $\mathrm{MgO}(5)$ & none & none & 24 & $0^{e}$ \\
\hline 28 & 1 & $\mathrm{SiO}_{2}(5)$ & none & none & 24 & $0^{e}$ \\
\hline 29 & 1 & $\mathrm{Al}_{2} \mathrm{O}_{3}(5)$ & none & none & 24 & $0^{e}$ \\
\hline 30 & 1 & $\mathrm{~K}_{2} \mathrm{CO}_{3}(5)$ & none & none & 24 & $0^{e}$ \\
\hline 31 & 1 & $\mathrm{CaO}(5)$ & none & none & 10 & $0^{e}$ \\
\hline 32 & 1 & $\mathrm{TiO}_{2}(5)$ & none & none & 24 & $0^{e}$ \\
\hline
\end{tabular}

${ }^{a}$ Conditions: Isobutyraldehyde $(1 \mathrm{mmol})$, in an oil bath at $120^{\circ} \mathrm{C} \cdot{ }^{b} 2 \mathrm{~mL}$ of the appropriate solvents were used under reflux conditions. ${ }^{c} 1 \mathrm{mmol}$ of the appropriate bases were used. ${ }^{d}$ Yields were determined by GC. ${ }^{e}$ Isobutyraldehyde $(1 \mathrm{mmol})$ was added drop wise. 


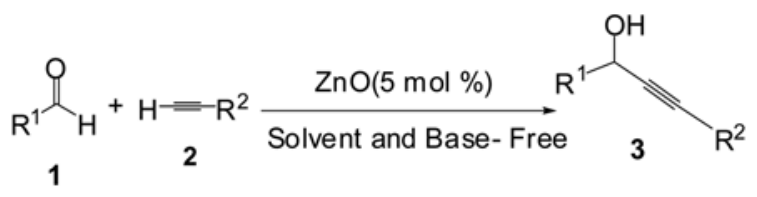

Scheme 1. Synthesis of propargylic alcohol derivatives catalyzed by $\mathrm{ZnO}$ under solvent-free condition.

technique. XRD pattern of solid $\mathrm{ZnO}$ is shown in Figure 1. The observed diffraction peaks in the recorded XRD patterns are in agreement with those of the JCPDS card 89-7102 for hexagonal $\mathrm{ZnO}$ and could be indexed to the hexagonal Wurtzite structure. No peaks of any other phase were detected. From the FWHM of diffraction lines, crystallite size is estimated employing Scherrer's formula. Average size is $>1 \mathrm{~mm}$.

In the next step, the commercially available solid $\mathrm{ZnO}(5$ mol \%) was mixed with benzaldehyde 1a (1 equiv) and phenylacetylene 2a ( 1 equiv) at $120{ }^{\circ} \mathrm{C}$ under solvent- and base-free conditions and 24 hour later, 1,3-diphenylprop-2yn-1-ol 3a was isolated in 50\% yield (Scheme 1, Table 1). When the amount of phenylacetylene was increased to two equivalents, the yield of the product was promoted to $98 \%$ and the reaction time decreased to 7 hours (entry 3 ). When the amount of phenylacetylene was less than 2 equiv, a competitive aldol condensation by-product was observed. Insteade of using excess amount of phenylacetylene, one simple way is the dropwise addition of aldehyde to alkyne.

Table 2. Synthesis of propargylic alcohols catalyzed by $\mathrm{ZnO}^{a}$

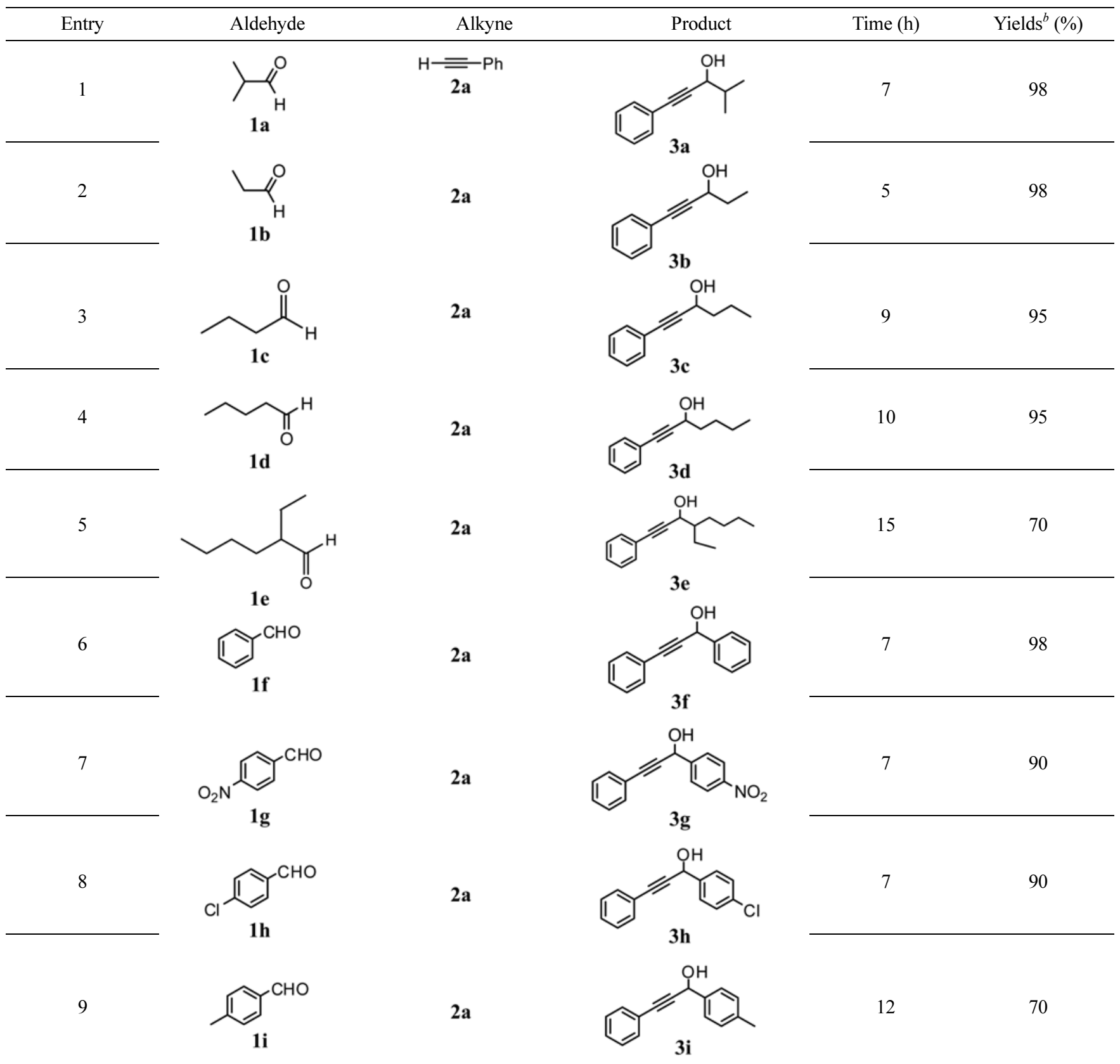


Table 2. Continued

\begin{tabular}{|c|c|c|c|c|c|}
\hline Entry & Aldehyde & Alkyne & Product & Time (h) & Yields $^{b}(\%)$ \\
\hline 10 & $1 \mathrm{j}$ & 2a & $3 \mathbf{j}$ & 7 & 92 \\
\hline 11 & & $2 a$ & & 12 & 75 \\
\hline 12 & & $2 a$ & & 7 & 95 \\
\hline 13 & & & & 12 & 85 \\
\hline 14 & & $2 \mathrm{a}$ & & 7 & 90 \\
\hline 15 & $1 \mathrm{a}$ & $2 b$ & & 12 & 70 \\
\hline 16 & $1 \mathrm{a}$ & & & 11 & 65 \\
\hline 17 & 1a & & & 13 & 60 \\
\hline 18 & $1 \mathrm{a}$ & & & 12 & 60 \\
\hline 19 & $1 \mathrm{~h}$ & & & 14 & 65 \\
\hline
\end{tabular}

${ }^{a}$ Conditions: aldehyde $(1 \mathrm{mmol})$, alkyne $(1 \mathrm{mmol})$, and $\mathrm{ZnO}(5 \mathrm{~mol} \%)$, in an oil bath at $120{ }^{\circ} \mathrm{C} .{ }^{b}$ Yields are determined by GC.

According to Table 1, entry 5, when iso-butyraldehyde was added in small portion to the phenylacetylene, the corresponding product was obtained in $98 \%$ yield after $7.5 \mathrm{~h}$. So to avoid aldol reactions, instead of reduplication of the phenylacetylene, slow addition of aldyhyde to alkyne was performed.

To obtain the optimized reaction conditions, several bases ( 1 equiv) and solvents ( $2 \mathrm{~mL}$ under reflux temperature) have been examined (Table 1, entries 6-19). This catalyst was not efficient in the presence of organic solvents such as methanol, acetonitrile, toluene, and strong bases such as $\mathrm{K}_{2} \mathrm{CO}_{3}$, $\mathrm{K}_{3} \mathrm{PO}_{4}, \mathrm{Cs}_{2} \mathrm{CO}_{3}$, and $\mathrm{Na}_{2} \mathrm{CO}_{3}$. Probably, $\mathrm{ZnO}$ surface contributes to the reaction so the solvents and bases may deactivate the catalyst and may also act as a contaminant, so it caused to decrease the reaction yields. Control experiment did not afford any products in the absence of $\mathrm{ZnO}$ even after 48 h (entry 20).

The quantitative variation of $\mathrm{ZnO}$ had performed influence 
on the reaction progress. The best amounts of nano $\mathrm{ZnO}$ were $20,15,10$, and $5 \mathrm{~mol} \%$, which afforded the desired product in 98\% yields (entries 21-24). However, when the amount of $\mathrm{ZnO}$ decreased, the yield of the product was also decreased but the reaction time increased (entries 25, 26). So, less than $5 \mathrm{~mol} \%$, ZnO was not an effective catalysis.

The yield of compound 3a has been examined by other organic catalysts (entries 27-32). We encountered with ineffective reactions, when $\mathrm{MgO}, \mathrm{SiO}_{2}, \mathrm{Al}_{2} \mathrm{O}_{3}, \mathrm{~K}_{2} \mathrm{CO}_{3}, \mathrm{CaO}$, and $\mathrm{TiO}_{2}$ were used under the same conditions. Therefore, the optimum reaction condition was found to be the dropwise addition of $1 \mathrm{mmol}$ aldehyde into $1 \mathrm{mmol}$ alkyne in the presence of $\mathrm{ZnO}(5 \mathrm{~mol} \%$ ) under solvent-free and without any additional bases. The experimental procedure is simple and straightforward.

With the above optimized conditions in hand, varieties of aromatic and aliphatic aldehydes with various terminal alkynes were screened to evaluate the scope of this reaction. We were pleased to find that all substrates were smoothly converted to the corresponding propargylic alcohols in good to excellent yields. The results are listed in Table 2 .

Aliphatic aldehydes showed high reactivity and afforded the corresponding propargylic alcohols in excellent yields. Both linear and branched aliphatic aldehydes (entries 1-5) undergo alkynylation with phenylacetylene in excellent yields. It is interesting to note that easily enolizable aldehydes (alkyl aldehydes $\mathrm{C}_{3}-\mathrm{C}_{5}$ ) produced alkynylated products 3a-e with $95-98 \%$ yields and no aldol condensation by products were formed. Benzaldehyde derivatives having both electron-donating and withdrawing substituents, 2-naphthaldehyde, and a heterocyclic aldehyde gave the products in good to excellent yields (entries 6-14).

We also checked on the reaction of aliphatic-substituted acetylenes such as 1-octyne, and alkynes $\mathbf{2 b}$-e with isobutyraldehyde under optimized conditions. Alkylacetylenes have low reactivity than aromatic acetylenes and many other catalysts or reagents reported previously did not work with these alkynes to produce propargylic alcohols. ${ }^{38}$ As it is shown in Table 2, entries 15-18, less reactive alkylacetylenes (much less acidic than 2a) were also applicable to the alkynylation of an aldehydes in good yields. In particular, alkynes 2b-e underwent the addition reaction with isobutyraldehyde 1a to furnish the corresponding propargylic alcohols in 60-65\% yields (entries 16-18), which is a costeffective process for the preparation of new compounds. As

Table 3. Reusability of $\mathrm{ZnO}$ in the synthesis of $\mathbf{3 a}^{a}$

\begin{tabular}{cccc}
\hline $\begin{array}{c}\text { Number } \\
\text { of use }\end{array}$ & $\begin{array}{c}\text { Used ZnO } \\
(\mathrm{g})\end{array}$ & $\begin{array}{c}\text { Recovered ZnO \% } \\
(\mathrm{g})\end{array}$ & $\begin{array}{c}\text { Yield of } \\
\mathbf{3 a}^{b}{ }^{b}\end{array}$ \\
\hline Fresh & 0.08 & $98(0.078)$ & 98 \\
1 & 0.078 & $98(0.076)$ & 98 \\
2 & 0.076 & $98(0.075)$ & 98 \\
3 & 0.076 & $95(0.072)$ & 92 \\
4 & 0.072 & $95(0.067)$ & 90 \\
\hline
\end{tabular}

${ }^{a}$ Conditions: isobutyraldehyde $(5 \mathrm{mmol})$, phenylaceylene $(10 \mathrm{mmol})$, in an oil bath at $120^{\circ} \mathrm{C} .{ }^{b}$ Isolated yields.

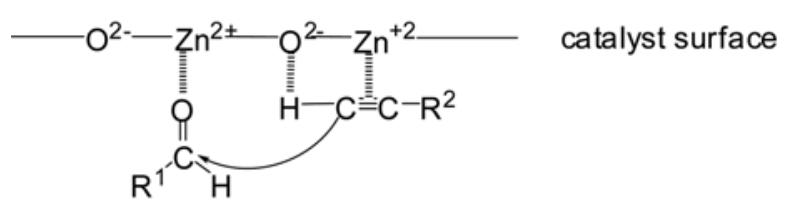

Scheme 2. Proposed mechanism for alkynylation via dual activation of both carbonyl and alkynes with $\mathrm{ZnO}$ as catalyst.

indicated above, solvent or base totally interrupted this alkynylation reaction, in this case to show the ability of $\mathrm{ZnO}$ as a good catalyst we apply this method to a solid alkyne and aldyhydes. As shown in Table 2 (entry 19) addition of 4chlorobenzaldehyde with alkyne 2 f afforded the corresponding alkynylated product $3 \mathrm{~s}$ in good yield. These substrates were melted under $120{ }^{\circ} \mathrm{C}$.

In order to investigate the recovery and reuse of the catalyst to improve that the process is heterogeneous, after the reaction was completed, the mixture was dilute with EtOAc and $\mathrm{ZnO}$ was removed by filtration. The catalyst after drying in an oven at $100{ }^{\circ} \mathrm{C}$ was reused for the same reaction. As shown in Table 3, the yields of 3a only decrease a little after the reuse of $\mathrm{ZnO}$ for four times.

Although the mechanism of $\mathrm{ZnO}$ catalyzed alkynylation of aldehydes has not yet been clarified, a mechanistic proposal for the role of $\mathrm{ZnO}$ as the catalyst is shown in Scheme 2. $\mathrm{ZnO}$ has Lewis acidic $\left(\mathrm{Zn}^{2+}\right)$ and basic sites $\left(\mathrm{O}^{2-}\right)$ and therefore could have dual activation. The oxygen of the carbonyl group is coordinated to $\mathrm{Zn}^{2+}$, resulting in the increased reactivity of aldehyde. The Lewis basic sites of $\mathrm{ZnO}\left(\mathrm{O}^{2-}\right)$ is coordinated to the hydrogen of terminal alkynes and followed by the nucleophilic attack to the carbonyl carbon of the aldehyde (Scheme 2).

\section{Conclusions}

In conclusion, we developed an efficient, new catalytic alkynylation of aldehydes using $\mathrm{ZnO}$ in solvent- and basefree conditions. The present method is complementary to the previously reported methods, and applicable to various aromatic and aliphatic aldehydes and alkynes. The cheap catalyst is commercially available and works well under solvent and base free conditions. The identity of the synthesis of propargyl chiral molecules and reaction mechanism remains obscure, and further work in this area is underway in our laboratory.

Acknowledgments. I gratefully acknowledge the support of this work by the Shiraz University Research Council.

\section{References}

1. Pu, L. Tetrahedron 2003, 59, 9873.

2. Stephane, G.; Aline, B.; Karen, P.; Annie, L.; Arnaud, H. Chem. Rev. 2006, 106, 2355.

3. Koyuncu, H.; Dogan, O. Org. Lett. 2007, 9, 3477.

4. Hudrlik, P. F.; Hudrlik, A. M. The Chemistry of the CarbonCarbon Triple Bond; Part 1. In Patai, S., Ed.; John Wiley and 
Sons: New York, 1978; p 256.

5. Cozzi, P. G.; Hilgraf, R.; Zimmermann, N. Eur. J. Org. Chem. 2004, 4095.

6. Rajaram, A. R.; Pu, L. Org. Lett. 2006, 8, 2019.

7. Lin, L.; Jiang, X. X.; Liu, W. X.; Qiu, L.; Xu, Z. Q.; Xu, J. K.; Chan, A. S. C.; Wang, R. Org. Lett. 2007, 9, 2329.

8. Li, Z.-B.; Liu, T.-D.; Pu, L. J. Org. Chem. 2007, 72, 4340.

9. Trost, B. M.; Weiss, A. H. Org. Lett. 2006, 8, 4461.

10. Tominaga, H.; Maezaki, N.; Yanai, M.; Kojima, N.; Urabe, D.; Ueki, R.; Tanaka, T. Eur. J. Org. Chem. 2006, 1422.

11. Treilhou, M.; Fauve, A.; Pougny, J.-R.; Promé, J.-C.; Veschambref, H. J. Org. Chem. 1992, 57, 3203.

12. Sierra, M. A.; Torres, M. R. J. Org. Chem. 2007, 72, 4213.

13. Johnson, W. S.; Brinkmeyer, R. S.; Kapoor, U. M.; Yarnell, T. M. J. Am. Chem. Soc. 1977, 99, 8341.

14. Chan, K. K.; Cohen, N. C.; Denoble, J. P.; Specian, A. C., Jr.; Saucy, G. J. Org. Chem. 1976, 41, 3497.

15. Gao, G.; Xie, R.-G.; Pu, L. Proc. Natl. Acad. Sci. U.S.A. 2004, $101,5417$.

16. Li, Z.-B.; Pu, L. Org. Lett. 2004, 6, 1065.

17. Xu, Z.; Chen, C.; Xu, J.; Miao, M.; Yan, W.; Wang, R. Org. Lett. 2004, 6, 1193.

18. Dahmen, S. Org. Lett. 2004, 6, 2113.

19. Liu, L.; Pu, L. Tetrahedron 2004, 60, 7427.

20. Kang, Y.-F.; Liu, L.; Wang, R.; Yan, W.-J.; Zhou, Y.-F. Tetrahedron: Asymmetry 2004, 15, 3155.

21. Cozzi, P. G. Angew. Chem., Int. Ed. 2003, 42, 2895.

22. Liu, L.; Wang, R.; Kang, Y.-F.; Chen, C.; Xu, Z.-Q.; Zhou, Y.-F.; Ni, M.; Cai, H.-Q.; Gong, M.-Z. J. Org. Chem. 2005, 70, 1084.

23. Brown, H. C.; Molander, G. A.; Singh, S. M.; Racherla, U. S. J. Org. Chem. 1985, 50, 1577.

24. Ahn, J. H.; Joung, M. J.; Yoon, N. M.; Oniciu, D. C.; Katritzky, A. R. J. Org. Chem. 1999, 64, 488.

25. Ahn, J. H.; Joung, M. J.; Yoon, N. M. J. Org. Chem. 1995, 60, 6173.

26. Hirao, T.; Misu, D.; Agawa, T. Tetrahedron Lett. 1986, 27, 933.

27. Imamoto, T.; Sugiura, Y.; Takiyama, N. Tetrahedron Lett. 1984, $25,4233$.

28. Frantz, D. E.; Fässler, R.; Carreira, E. M. J. Am. Chem. Soc. 2000, $122,1806$.

29. Frantz, D. E.; Fässler, R.; Tomooka, C. S.; Carreira, E. M. Acc. Chem. Res. 2000, 33, 373.

30. Anand, N. K.; Carreira, E. M. J. Am. Chem. Soc. 2001, i123, 9687.
31. Fässler, R.; Tomooka, C. S.; Frantz, D. E.; Carreira, E. M. Proc. Natl. Acad. Sci. U.S.A. 2004, i101, 5843.

32. Frantz, D. E.; Fässler, R.; Carreira, E. M. J. Am. Chem. Soc. 1999, 121,11245

33. Yamaguchi, M.; Hayashi, A.; Minami, T. J. Org. Chem. 1991, 56, 4091.

34. Yamaguchi, M.; Hayashi, A.; Hirama, M. Chem. Lett. 1992, 2479.

35. Han, Y.; Huang, Y.-Z. Tetrahedron Lett. 1995, 36, 7277.

36. Jiang, B.; Si, Y.-G. Tetrahedron Lett. 2002, 43, 8323.

37. Harada, S.; Takita, R.; Ohshima, T.; Matsunaga, S.; Shibasaki, M. Chem. Commun. 2007, 948.

38. Takita, R.; Fukuta, Y.; Tsuji, R.; Ohshima, T.; Shibasaki, M. Org Lett. 2005, 7, 1363.

39. Tsuji, R.; Yakura, K.; Ohshima, T.; Shibasaki, M. J. Am. Chem. Soc. 2005, 127, 13760.

40. Sakai, N.; Hirasawa, M.; Konakahara, T. Tetrahedron Lett. 2003, $44,4171$.

41. Sakai, N.; Kanada, R.; Hirasawa, M.; Konakahara, T. Tetrahedron 2005, 61, 9298.

42. Natalia, M.-V.; Julia, K.; Ilan, M. Synthesis 2000, 917.

43. Kazuhiko, T.; Tooru, K.; Shigeki, N.; Koichiro, O.; Hitosi, N. Tetrahedron Lett. 1985, 45, 5585.

44. Xuefeng, J.; Hongwei, Y.; Ling, F.; Chengjian, Z. Tetrahedron Lett. 2008, 49, 1370.

45. Srihari, P.; Singh, V. K.; Bhunia, D. C.; Yadav, J. S. Tetrahedron Lett. 2008, 49, 7132 .

46. Gu, C. Z.; Li, Q. R.; Yin, H. Chin. Chem. Lett. 2005, 16, 1573.

47. Justicia, J.; Sancho-Sanz, I.; Alvarez-Manzaneda, E.; Oltra, E.; Cuerva, J. M. Adv. Synht. Catal. 2009, 351, 2295.

48. Loh, T. P.; Lin, M.-J.; Tan, K.-L. Tetrahedron Lett. 2003, 44, 507.

49. (a) Sharghi, H.; Hosseini Sarvari, M. Synthesis 2002, 8, 1057. (b) Hosseini-Sarvari, M.; Sharghi, H. J. Org. Chem. 2004, 69(20), 6953. (c) Hosseini-Sarvari, M.; Synthesis 2005, 5, 787. (d) Hosseini-Sarvari, M.; Sharghi, H. Tetrahedron 2005, 61, 10903. (e) Hosseini-Sarvari, M.; Sharghi, H. J. Org. Chem. 2006, 71, 6652. (f) Hosseini-Sarvari, M.; Sharghi, H. Phos. Silicon. Sulf. 2007, 182, 2125. (g) Hosseini-Sarvari, M. Synth. Commun. 2008, i6, 832. (h) Hosseini-Sarvari, M. Act. Chim. Slov. 2008, 440. (i) Hosseini-Sarvari, M.; Sharghi, H.; Etemad, S. Helv. Chim. Acta 2008, 91, 715. (j) Hosseini-Sarvari, M.; Catalysis Lett. 2011, 114, 347. (k) Hosseini-Sarvari, M.; Mardaneh, Z. Bul. Chem. Soc. Jpn 2011, 84,778 . 